https://doi.org/10.11646/zootaxa.4303.1.5

http://zoobank.org/urn:lsid:zoobank.org:pub:E0EFFC3A-8364-4559-9B2C-D252FC7C627E

\title{
Type material housed in the Carcinological Collection of the Museo de La Plata, Argentina
}

\author{
ANALÍA R. DIAZ ${ }^{1,2}$, VICTOR HUGO MERLO ÁLVAREZ ${ }^{2} \&$ CRISTINA DAMBORENEA ${ }^{2,3}$ \\ ${ }^{1}$ Instituto de Limnología Dr. Raúl A. Ringuelet (CCT-CONICET La Plata, FCNyM-UNLP), Boulevard 120 y 62 (1900) La Plata, \\ Provincia de Buenos Aires, Argentina \\ ${ }^{2}$ División Zoología Invertebrados, Museo de La Plata, FCNyM-UNLP, Paseo del Bosque s/n, 1900 La Plata, Argentina. \\ E-mail: analiadiaz@ilpla.edu.ar; hugmerlo@fcnym.unlp.edu.ar; cdambor@fcnym.unlp.edu.ar \\ ${ }^{3}$ CONICET
}

\begin{abstract}
The Carcinological Collection of División Zoología Invertebrados, Museo de La Plata (FCNyM-UNLP) includes of 110 type lots of 42 species. A list of types of the crustacean orders Anostraca ( 8 species), Diplostraca (5 species), Arguloida (3 species), Cyclopoida (1 species), Poecilostomatoida (8 species), Siphonostomatoida (2 species), Podocopida (4 species), Amphipoda (2 species), Isopoda (2 species), Mysida (2 species) and Decapoda (5 species) is presented. Species names are listed in systematical order, including type locality, collection number, current taxonomic status and original bibliographic reference. For parasite and commensal species, type host and site of infection are also provided.
\end{abstract}

Key words: Crustacea, catalogue, type material, Museo de La Plata

\section{Introduction}

Natural history collections play an important role in the study and understanding of biodiversity. For such knowledge, type specimens are the base units of zoological nomenclature. The Invertebrate Collection of Museo de La Plata (MLP) of the Facultad de Ciencias Naturales y Museo, Universidad Nacional de La Plata, Argentina, is a significant resource of information on the invertebrate (excluding insects) biodiversity of Argentina. One of those collections is the Carcinological Collection, which consists of approximately 2,200 records. Of these crustacean records, $90 \%$ are from Argentina; approximately $65 \%$ are from inland waters (streams, rivers, lakes and ponds) and $35 \%$ are from marine and brackish environments. Crustacean groups with the highest number of records belong to Decapoda, followed in importance by Isopoda, Anostraca, Amphipoda and other records pertaining to Cirripedia, Branchiura and Copepoda. The province of Buenos Aires is the region best represented, with the largest number of lots, followed by Chubut, Tierra del Fuego, Santa Cruz and Río Negro.

The foundation of the collection was laid by Max Birabén and Raúl A. Ringuelet. The oldest specimens are from 1896-1897 and were collected by Juan Durione, Fernando Lahille and Miguel Fernandez. Since then, the collection increased thanks to researchers, students and citizen scientists. Researchers like G. Bond-Buckup, E. C. Lopretto, I. I. César, J. T. Timi and J. A. Etchegoin and others made important contributions to the collection while studying e.g. Aeglidae, Argulidae, free living and parasitic Copepoda, fish parasites, Trichodactilidae crabs, Sergestidae, and other shrimp. The collection also holds 550 zooplankton samples, including Copepoda and Cladocera from different localities of Argentina. Type material of 42 crustacean species, represented by 110 specimen lots, amounts to $5 \%$ of all records in the collection.

All records are digitized in a database using the software Access 2007 (Microsoft). Approximately $50 \%$ of the records are identified at generic or specific level and are currently available on GBIF sites (dataset Facultad de Ciencias Naturales y Museo).

The purpose of this publication is to provide a complete catalog of the type material currently deposited in the Carcinological Collection of the Museo de La Plata, Argentina. 


\section{Material and methods}

The specimens are stored in glass containers conserved in 70\% ethanol, but some of them fixed in Formalin. Other specimens are stored in permanent and semi-permanent slide preparations (especially for microcrustaceans). There are also dry microscopic preparations and many whole dried decapods. The Collection is organized in lots. Each lot is a group of specimens from the same locality, the same date and collector. Wet lots are housed in a storage room specially equipped for wet conservation, shared with other Invertebrate Zoology Collections. This storage room has a forced air system for ventilation, an electrical system protected against vapors and fire extinguishers. The non-wet preparations are arranged in another storage room on wooden shelves. All the material is available for consultation. Unless otherwise stated, all the material is in good condition.

Each entry includes:

1 The name of the taxon in the original combination with its author(s) and year of publication. Asterisk $(*)$ denotes type species of the genus.

2 Type locality including country, province or state, department, specific locality, and coordinates.

3 Type host for parasite or commensal species, indicating species, author(s), and principal taxonomic group in brackets.

4 Site of infection in parasite or commensal species.

5 Type status (eg. holotype/syntype/paratype), the number and sex of specimens, MLP-Cr catalog number, date and name of collectors. The type categories follow articles 73-75 of the ICZN (1999).

6 Current taxonomic status, followed by revision history when applicable.

7 Additional type holdings at other repositories.

8 Remarks section when necessary.

9 Reference(s) includes publications with the original descriptions and those that mention type specimens of the MLP Carcinological Collection.

Cases where date of collection, locality and collector are not specified, this information is not available. Notwithstanding our efforts in finding type specimens that were cited in the literature as having been deposited in MLP Carcinological Collection, some of them have not been found up to now.

The classification follows Martin \& Davis (2001).

Abbreviations: AMG: Albany Museum, Grahamstown, South Africa; BMNH: British Museum Natural History, London, UK; CC-FC: Colección de Crustáceos. Facultad de Ciencias, Universidad de La República, Montevideo, Uruguay; FML: Miguel Lillo Foundation, Instituto de Biología, Tucumán, Argentina; IPCAS: Institute of Parasitology, Academy of Sciences of the Czech Republic, Èeské Budijovice, Czech Republic; MACNIn: Museo Argentino de Ciencias Naturales "Bernardino Rivadavia", Buenos Aires City, Argentina; MLP-Cr: Carcinological Collection, Museo de La Plata, Facultad de Ciencias Naturales y Museo, Universidad Nacional de La Plata, Argentina; MNRJ: Museo Nacional de Rio du Janeiro, Rio de Janeiro, Brazil; NMA: National Museum of Australia, Canberra, Australia; SM: Sars Museum, Oslo, Norway; USNM: National Museum of Natural History, Washington DC, USA.

\section{Results}

Class BRANCHIOPODA Latreille, 1817

Subclass Sarsostraca Tasch, 1969

Order Anostraca Sars, 1867

Family Branchinectidae Daday, 1910 
Type locality. Argentina, Córdoba Province, Pampa de Achala County $\left(30^{\circ} 33^{\prime} \mathrm{S}-63^{\circ} 31^{\prime} \mathrm{W}\right)$.

Holotype. ${ }^{\lambda}$, MLP-Cr 2; 1983-06-18. Collector: César, I.

Paratypes. MLP-Cr 24/2, same locality as the type, 1943-02-25. Collector: Birabén, M.; MLP-Cr 152/1, same locality as type, 1981-02-28. Collector: César, I.; MLP-Cr 25/3, Argentina, Córdoba Province, Los Gigantes (31 $\left.{ }^{\circ} 23^{\prime} 60^{\prime \prime S}-64^{\circ} 46^{\prime} 60^{\prime \prime} \mathrm{W}\right)$, Collector: Birabén, M.; MLP-Cr 7/3, Argentina, Buenos Aires City, Bañado de Flores, near Riachuelo, 1950-02-16, Collector: Bachman, A.

Current status. Valid species.

Remarks. Other material examined, collected in "Los Gigantes" (Córdoba Province) and "Bañado Las Flores" (Buenos Aires City) here assigned as paratypes.

Reference. César (1985).

\section{Branchinecta leonensis César, 1987}

Type locality. Argentina, Santa Cruz Province, Cerro León County (48²1'07'"S-66³8'50"W)

Holotype. J, MLP-Cr3, 1948-03-07. Collector: Birabén, M.

Paratypes. + , MLP-Cr 4/2, same locality as type, 1948-03-07, Collector: Birabén, M.

Current status. Valid species.

Reference. César (1987a).

\section{Branchinecta palustris Birabén, 1946}

Type locality. Argentina. Río Negro Province, La Escondida lagoon ( $\left.40^{\circ} 18^{\prime} \mathrm{S}-65^{\circ} 58^{\prime} \mathrm{W}\right)$.

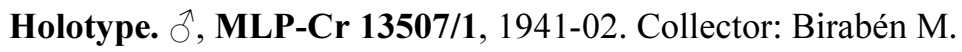

Allotype. $q$, MLP-Cr 13507/2.

Paratype ${ }^{\top}$ MLP-Cr 13507/3, same locality, date and collector as type.

Current status. Valid species.

Additional types. Paratypes. $\delta$ and $q$ at MACN-In.

Reference. Birabén (1946).

\section{Branchinecta papillosa Birabén, 1946}

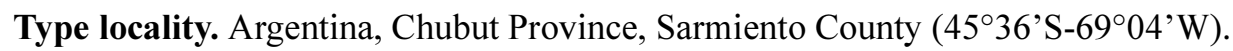

Holotype. ${ }^{\lambda}$, MLP-Cr 13504/1, 1938-02-15.

Allotype. + , MLP-Cr 13504/2.

Paratypes. $\delta$ and $\odot$, MLP-Cr 13504/3.

Current status. Valid species

Additional types. Paratypes. $\delta$ and $q$ at MACN-In

Remarks. Type material lost.

Reference. Birabén (1946).

\section{Branchinecta santacrucensis César, 1987}

Type locality. Argentina, Santa Cruz Province, Coyle County near Güer Aike (51 $\left.{ }^{\circ} 37^{\prime} \mathrm{S}-69^{\circ} 37^{\prime} \mathrm{W}\right)$.

Holotype. $\overbrace{}^{\lambda}$, MLP-Cr 4/1, 1939-03-15. Collector: Birabén M.

Paratypes. 7 and 20 , MLP-Cr 90/1, same locality, date and collector as type.

Current status. Junior synonym of Branchinecta granulosa Daday, 1902 according to Cohen (1995).

Reference. César (1987b), Cohen (1995) 
Type locality. Argentina, Santa Cruz Province, pools near Tur Lake $48^{\circ} 54^{\prime} \mathrm{S}-72^{\circ} 36^{\prime} \mathrm{W}$

Holotype. ${ }^{\lambda}$, MLP-Cr 13500/1, 1936-03-02. Collector: Birabén M.

Paratypes. $q$ (allotype), MLP-Cr 13500/2; 6 and 20 , MLP-Cr 13500/3, same locality, date and collector as type.

Current status. Valid species.

Additional types. Paratype $q$ deposited at MACN-In.

Reference. Birabén (1946).

\section{Branchinecta valchetana Cohen, 1981}

Type locality. Argentina, Río Negro Province, Provincial Route 251, between General Conesa and San Antonio Oeste (402 $\left.27^{\prime} \mathrm{S}-64^{\circ} 37^{\prime} \mathrm{W}\right)$.

Paratypes. 2 and $2 \circ$, MLP-Cr 157, from the same locality as type, 1974-07. Collector: Bachmann, A.

Current status. Valid species.

Additional types. Holotype. $\lesssim$ MACN-In 30273. Allotype. + MACN-In 30275. Paratypes. $2 \uparrow$ MACN-In 30274 and 2 + MACN-In 30276.

Reference. Cohen (1981).

Family Thamnocephalidae Simon, 1886

Phallocryptus salinicola Birabén, 1951

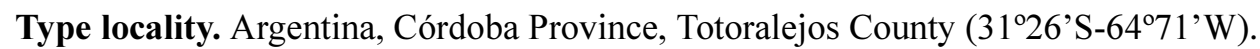

Syntype. ${ }^{\lambda}$, MLP-Cr 52/1 and ${ }^{\text {, }}$, MLP-Cr 52/2, 1950-03-08. Collector: Birabén M.

Current status. Junior synonym of Phallocryptus uriga (Smirnov, 1948) according to Belk \& Brtek (1995).

Additional types. Syntypes MACN-In 9411, and MACN-In 32039.

Reference. Birabén (1951), Belk \& Brtek (1995).

Subclass Phyllopoda Preuss, 1951

Order Diplostraca Gerstaecker, 1866

Suborder Spinicaudata Linder, 1945

Family Limnadiidae Baird, 1849

Limnadia sanitaguensis César, 1991

Type locality. Argentina, Santiago de Estero Province, Salinas Grande County (300'ㅅ-

$\left.65^{\circ} 13^{\prime} \mathrm{W}\right)$.

Holotype. đ̊, MLP-Cr 26886/1, 1959-12-14. Collector: Birabén M.

Paratypes. MLP-Cr 26886/2, same locality, date and collector as type.

Current status. Valid species.

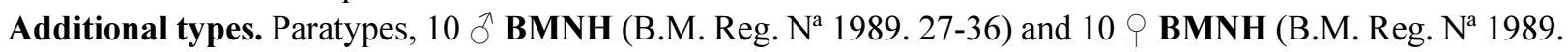
27-46).

Reference. César (1991). 
Suborder Cladocera Latreille, 1829

Infraorder Anomopoda Stebbing, 1902

Family Chydoridae Stebbing, 1902

Pleuroxus macquariensis Frey, 1993

Type locality. Australia, Macquarie Island ( $\left.54^{\circ} 30^{\prime} \mathrm{S}-158^{\circ} 57^{\prime} \mathrm{E}\right)$.

Paratype. 1 parthenogenetic + , MLP-Cr27164, 1963. Collector: Jolly, V., same locality as type.

Current status. Valid species

Additional types. Holotype. NMA P40965. Paratypes. 1 Parthenogenetic $q$ respectively in USNM, BMNH, AMG, NMA, SM and Frey's personal collection in Bloomington.

Reference. Frey (1993).

\section{Pleuroxus paraplesius Frey, 1993}

Type locality. Argentina, Santa Cruz Province, Monte Dinero, on road from Cabo Virgenes to Rio Gallegos (52 $\left.10^{\circ} \mathrm{S}-69^{\circ} 03^{\prime} \mathrm{W}\right)$.

Holotype. 1 mature parthenogenetic ${ }_{+}$, MLP-Cr 27165, 1989-01-28. Collector: Frey, D.G.

Allotype. $\hat{\delta}$, MLP-Cr 27166 and $\hat{\partial}$ and $\phi$, MLP-Cr 27167, same locality, date and collector as type.

Current status. Valid species.

Additional types. Paratypes. 2 parthenogenetic $q$ respectively in AMG, NMA, USNM, BMNH and SM.

Reference. Frey (1993).

\section{Pleuroxus varidentatus Frey, 1993}

Type locality. Argentina, Santa Cruz Province, Marshy channel in Punta Bandera on the southern shore of Lago Argentino (52 $\left.18^{\circ} \mathrm{S}-72^{\circ} 46^{\prime} \mathrm{W}\right)$.

Holotype. 1 parthenogenetic 9 , MLP-Cr 27168, 1989-01-20, Collector: Frey, D.G.

Paratype. + , MLP-Cr 27169, same locality, date and collector as type.

Current status. Valid species.

Additional types. Paratypes $\mathbf{2}$ parthenogenetic $q$, mounted on the same slide in glycerol jelly and $\mathbf{5}$ mature $q$ in a 1:1 mixture of 3\% formaldehyde and glycerol AMG, NMA, USNM, BMNH and SM.

Reference. Frey (1993).

\section{Lynceus mallinensis Pessacq, Epele \& Rogers, 2011}

Type locality. Argentina, Chubut Province, Cholila, State Route 15, $28 \mathrm{~km}$ east of Cholila town, wetland by the side of the road $\left(42^{\circ} 21^{\prime} 43.3^{\prime \prime} \mathrm{S}-71^{\circ} 08^{\prime} 59.8^{\prime \prime} \mathrm{W}\right)$.

Holotype. $\overbrace{}^{\lambda}$, MLP-Cr 26724, 2006-12. Collectors: Epele, L., Miserendino, L. and Pessacq, P.

Allotype. $\circ$, MLP-Cr 26725.

Paratypes, 11 and $5 \AA$, MLP-Cr 26726, same locality, date and collector as type.

Current status. Valid species.

Additional types. $1 \hat{\delta}$ and $\mathbf{2} \odot$, same locality, date and collector as type, DCR-782, personal collection of D. Christopher Rogers.

Reference. Pessacq et al. (2011). 
Class MAXILLOPODA Dahl 1956

Subclass Branchiura Thorell, 1864

Order Arguloida Yamaguti, 1963

Family Argulidae Leach, 1819

Argulus paranensis Ringuelet, 1943

Type host. Salminus brasiliensis (Cuvier, 1816) (Characiformes:Characidae)

Site of infection. Body surface.

Type locality. Argentina,Entre Ríos Province, Paraná City, Las Conchas stream ( $\left.31^{\circ} 44^{\prime} \mathrm{S}-60^{\circ} 12^{\prime} \mathrm{W}\right)$.

Holotype. ㅇ, MLP-Cr 41, Collector: Allen, C.

Current status. Valid species.

Reference. Ringuelet (1943).

\section{Argulus patagonicus Ringuelet, 1943}

Type host. Percichthys trucha (Valenciennes 1833) (Perciformes: Percichthyidae)

Site of infection. Body surface.

Type locality. Argentina, Rio Negro Province, Pelegrini Lake (3841'S-6759' W).

Holotype. + , MLP-Cr 128, 1935-07-17. Collector: Mac Donagh, E. J.

Paratypes. $4 \delta$ and $2 \circ$, MLP-Cr 129, same locality, host, date and collector as type.

Current status. Valid species.

Reference. Ringuelet (1943).

\section{Argulus pestifer Ringuelet, 1948}

Type host. Salminus brasiliensis (Cuvier, 1816) (Characiformes: Characidae).

Site of infection. Body surface.

Type locality. Argentina, Entre Ríos Province.

Syntype. ㅇ, MLP-Cr 40, 1940-01. Collector: Castillos, S.

Current status. Valid species.

Reference. Ringuelet (1948a).

Subclass Copepoda Milne-Edwards, 1840

Order Cyclopoida Burmeister, 1834

Family Ozmanidae Ho \& Thatcher, 1989

Ozmana huarpium Gamarra-Luques \& Castro-Vazquez, 2004

Type host. Pomacea canaliculata (Lamarck, 1822) (Mollusca: Ampullariidae).

Site of infection. Haemocoel, mantle cavity, ctenidium and penis sheath's groove.

Type locality. Argentina, Buenos Aires city, Rosedal lake (34은 $\left.34^{\prime} \mathrm{S}-58^{\circ} 24^{\prime} \mathrm{W}\right)$.

Paratypes. 10 $\uparrow$, MLP-Cr 25806; 10 ovigerous with eggs, MLP-Cr 25807; 10 , MLP-Cr 25808, same locality and host as type, 2004-03. Collector: Gamarra-Luques, C.D.

Current status. Valid species. 
Additional types. Holotype. MACN-In 36047; Allotype. MACN-In 36048; Paratypes. MACN-In 36049/ $36050 / 36051$.

Reference. Gamarra-Luques et al. (2004).

\section{Order Poecilostomatoida Thorell, 1859}

Family Bomolochidae Claus, 1875

Nothobomolochus cresseyi Timi \& Sardella, 1997

Type host. Engraulis anchoita Hubbs \& Marini, 1935 (Clupeiformes: Engraulidae)

Site of infection. Gills.

Type locality. Argentina, Buenos Aires coastal area (36 $52^{\circ}$ 'S $-55^{\circ} 28^{\prime} \mathrm{W}$ ).

Holotype. , MLP-Cr 26072, 1995-09. Collector: Timi, J.

Paratypes. 2 , MLP-Cr 26073, 1994-10, same locality, host and collector as type.

Current status. Valid species.

Remarks. 2 paratypes, IPCAS.

Reference. Timi \& Sardella (1997).

Family Chondracanthidae Milne Edwards, 1840

Acanthochondria helicoleni Cantatore \& Timi, 2010

Type host. Helicolenus lahillei Norman, 1937 (Scorpaeniformes: Sebastidae)

Site of infection. Branchiostegal pocket and gill arch.

Type locality. Argentinean-Uruguayan Common Fishing ( $\left.36^{\circ} 23^{\prime}-36^{\circ} 49^{\prime} \mathrm{S}, 54^{\circ} 00-54^{\circ} 11^{\prime} \mathrm{W}\right)$

Holotype. + , MLP-Cr 26533, 2008-02. Collector: Cantatore, D.

Allotype. $\delta^{\lambda}$, MLP-Cr 26534, same locality, host, date and collector as type.

Paratype. $2+$ with 1 t attached, MLP-Cr 26535, same locality, host, date and collector as type.

Current status. Valid species.

Reference. Cantatore \& Timi (2010).

Acanthochondria lilianae Cantatore, Lanfranchi \& Timi, 2011

Type host. Patagonotothen ramsayi (Regan, 1913) (Perciformes: Nototheniidae).

Site of infection. Inner surface of operculum.

Type locality. Argentina, Patagonian Shelf ( $\left.42^{\circ} 48^{\prime} \mathrm{S}-60^{\circ} 63^{\prime} \mathrm{W}\right)$.

Holotype. + , MLP-Cr 26731, 2007-11.

Allotype. ${ }^{3}$, MLP-Cr 26732, same locality and date as type.

Paratype. $2+$ with 1 attached ${ }^{\lambda}$, MLP-Cr 26733, same locality and date as type.

Current status. Valid species.

Reference. Cantatore et al. (2011).

\section{Acanthochondria sagita Alarcos \& Timi, 2011}

Type host. Xystreurys rasile (Jordan, 1890) (Pleuronectiformes: Paralichthyidae).

Site of infection. Gill rakers.

Type locality. Argentina, Coastal waters of Necochea (38 $\left.36^{\circ} \mathrm{S}-58^{\circ} 42^{\prime} \mathrm{W}\right)$.

Holotype. 9 , MLP-Cr 26558, 2009-12. Collector Alarcon, M. J. 
Allotype. $\lesssim$, MLP-Cr 26559, same locality, host, date and collector as type; $2 \uparrow$ with $1 \precsim$ attached, MLP-Cr 26560, same locality, host, date and collector as type.

Current status. Valid species.

Additional types. Paratypes. 2 sex, IPCAS (Cat.No. Cr-12).

Reference. Alarcos \& Timi (2011).

\section{Acanthochondria serrani Braicovich \& Timi, 2009}

Type host. Serranus auriga (Cuvier 1829) (Perciformes: Serranidae)

Site of infection. Inner side of operculum.

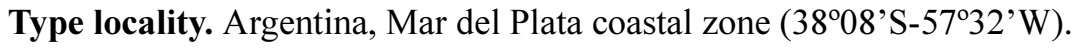

Holotype. + , MLP-Cr 26209.

Allotype. $\curvearrowright$, MLP-Cr 26210.

Paratypes. 2 q each with 1 đึ attached, MLP-Cr 26211. May-July, October 2007, January, March-June 2008.

Current status. Valid species.

Additional types. Paratypes, $1 \uparrow$ with $1 \lesssim$, IPCAS (Cat.No. Cr-10).

Reference. Braicovich \& Timi (2009).

\section{*Argentinochondria patagonensis Etchegoin, Timi \& Sardella, 2003}

Type host. Genypterus brasiliensis Regan, 1903 (Ophidiiformes: Ophidiidae).

Site of infection. Inner side of operculum, with head and neck buried into the host tissue.

Type locality. Argentina, San Jorge Gulf, Patagonia $\left(47^{\circ} \mathrm{S}-67^{\circ} 30^{\prime} \mathrm{W}\right)$.

Holotype. + , MLP-Cr 5102, 1993-10.

Allotype. $\hat{\sigma}$, MLP-Cr 5103, same locality, host and date as type.

Paratypes. + , $\hat{\sigma}$, MLP-Cr5104, 2 with attached $\hat{\sigma}$, same locality, host and date as type.

Current status. Valid species.

Reference. Etchegoin et al. (2003).

\section{Blias marplatensis Timi, Etchegoin \& Lanfranchi, 2004}

Type host. Prionotus nudigula Ginsburf 1950 (Scorpaeniformes: Triglidae).

Site of infection. Inner side of operculum.

Type locality. Argentina, Buenos Aires Coastal area of Mar del Plata (38 $08^{\prime}$ S $-57^{\circ} 32^{\prime}$ W).

Holotype. 9 , MLP-Cr 25809, 2004-05. Collector: Lanfranchi, A.

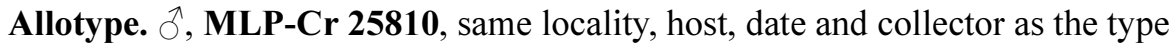

Paratypes. $3 q \circ$, MLP-Cr 25811, same locality, host, date and collector as type.

Current status. Valid species.

Reference. Timi et al. (2004).

\section{Family Philichthyidae Vogt, 1877}

\section{Colobomatus sudatlanticus Pereira, Timi, Lanfranchi \& Luque, 2012}

Type host. Mullus argentinae Hubbs \& Marini, 1933 (Perciformes: Mullidae).

Site of infection. Pores of the cephalic sensory system and nostrils of hosts.

Type locality. Brazil, Santa Catarina State, Florianópolis $\left(27^{\circ} 10^{\prime} \mathrm{S}-48^{\circ} 35^{\prime} \mathrm{W}\right) .2010-08$.

Paratypes. 5 , MLP-Cr 26759, 2011-08, same host as type, Argentina. Coastal waters of Mar del Plata $\left(38^{\circ} 08^{\prime} \mathrm{S}-57^{\circ} 32^{\prime} \mathrm{W}\right)$. Collector: Timi, J. 
Current status. Valid species.

Additional types. Holotype. $\odot$, MNRJ 23317. Paratypes. 10 , MNRJ 23318-23320.

Reference. Pereira et al. (2012).

\section{Order Siphonostomatoida Thorell, 1859}

Family Lernaeopodidae Milne Edwards, 1840

\section{Parabrachiella spinicephala Ringuelet, 1945}

Type host. Pinguipes fasciatus Jennyns, 1840 (Perciformis: Pinguipedidae)

Site of infection. Pectoral fin.

Type locality. Argentina, Buenos Aires, Mar del Plata County $\left(38^{\circ} 05^{\prime} \mathrm{S}-57^{\circ} 32^{\prime} \mathrm{W}\right)$.

Syntype. 2 MLP-Cr 26922, 1929-11.

Current status. Valid species. Neobrachiella spinicephala (Ringuelet, 1945), new combination according to Etchegoin et al. (2006).

Reference. Ringuelet (1945), Etchegoin et al. (2006).

Family Lernanthropidae Kabata, 1979

Lernanthropus cynoscicola Timi \& Etchegoin, 1996

Type host. Cynoscion striatus (Cuvier, 1829) (Perciformes: Sciaenidae).

Site of infection. Gills.

Type locality. Argentina, Buenos Aires Province coastal area $\left(38^{\circ} 05^{\prime} \mathrm{S}-57^{\circ} 32^{\prime} \mathrm{W}\right)$.

Holotype. + , MLP-Cr 26918, 1994-08. Collector: Timi, J.

Allotype. $\widehat{\alpha}$, MLP-Cr 26919, same locality, host, date and collector as type.

Paratypes. 5 , MLP-Cr 26920, same locality, host, date and collector as type.

Current status. Valid species.

Additional types. Paratype, IPCAS (Collector No-Cru-1).

Reference. Timi \& Etchegoin (1996).

\section{Class OSTRACODA Latreille, 1802}

\section{Subclass Podocopa Sars, 1886}

\section{Order Podocopida Sars, 1866}

\section{Family Candonidae Kaufmann, 1900}

Keysercypria ivanae Díaz \& Lopretto, 2011

Type locality. Argentina, Buenos Aires Province, Magdalena County, Atalaya ( $35^{\circ} 02^{\prime}$ 'S$\left.57^{\circ} 32^{\prime} \mathrm{W}\right)$.

Holotype. $\widehat{~}$, MLP-Cr 26498, 2008-12. Collector: Díaz, A.

Allotype.. + ,MLP-Cr 26499, same locality, date and collector as type

Paratypes. $1 \propto$ and $5 \hat{\partial}$, MLP-Cr 26500, same locality, date and collector as type.

Current status. Valid species.

Reference. Díaz \& Lopretto (2011). 
*Argentocypris sara Díaz \& Martens, 2014

Type locality. Argentina, Neuquén Province, Laguna Blanca $\left(39^{\circ} 02^{\prime} \mathrm{S}-70^{\circ} 21^{\prime} \mathrm{W}\right)$.

Holotype. ${ }^{\lambda}$, MLP-Cr 26779, 2005-12-11. Collector: Marione, M. C

Allotype. + , MLP-Cr 26780, same locality, date and collector as type.

Paratypes. 2 and 9 , MLP-Cr 26781, same locality, date and collector as type.

Current status. Valid species.

Reference. Díaz \& Martens (2014).

\section{Cypriconcha hypsophila Díaz \& Lopretto, 2009}

Type locality. Argentina, Catamarca Province, Tinogasta, Los Aparejos (28 $03^{\circ}$ 'S-67'33' W).

Holotype. ${ }^{\lambda}$, MLP-Cr 26137, 2005-01-04. Collector: Locascio, C.

Allotype. + , MLP-Cr 26138, same locality, date and collector as type.

Paratypes. 26 and $16 \AA$, MLP-Cr 26139, same locality, date and collector as type.

Current status. Valid species.

Reference. Díaz \& Lopretto (2009).

\section{Family Cytherideidae Sars, 1925}

Cyprideis salebrosa hartmanni Ramirez, 1967

Type locality. Argentina, Buenos Aires Province, La Plata ( $\left.34^{\circ} 55^{\prime} \mathrm{S}-57^{\circ} 57^{\prime} \mathrm{W}\right)$.

Holotype. MLP-Cr 100, 1960-02. Collector: Ramírez, F.

Current status. Synonym of Cyprideis hartmanni Ramírez, 1967.

Remarks. Material lost.

Reference. Ramírez (1967).

Class MALACOSTRACA Latreille, 1802

Subclass Eumalacostraca Grobben, 1892

Superorder Peracarida Calman, 1904

Order ISOPODA Latreille, 1817

Suborder Asellota Latreille, 1802

Family Paramunnidae Vanhöffen, 1914

Austrosignum fuegiae Doti \& Roccatagliata, 2005

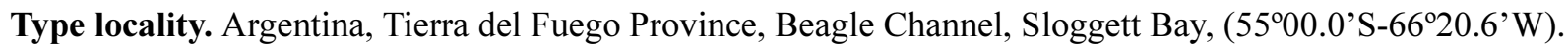

Holotype. ㅇ, MLP-Cr 25812, 2001-02. Collector: Zelaya, D.

Paratypes. $20 \uparrow$ and $40 \AA$, MLP-Cr 25813, same locality, date and collector as type.

Additional types. Paratypes MACN-In 36246, MACN-In 36245.

Current status. Valid species. New combination Advenogonium fuegiae (Doti \& Roccatagliata, 2005) and type species of Advenogonium according to Just \& Wilson (2007).

Reference. Doti \& Roccatagliata (2005), Just \& Wilson (2007). 
Suborder Cymothoida Wägele, 1989

Infraorder Epicaridea Brandt \& Poore (2003)

Family Bopyridae Rafinesque, 1815

Probopyrus ringueleti Verdi \& Schuldt, 1988

Type host. Palaemonetes argentinus Nobili, 1901 (Caridea, Palaemonidae)

Site of infection. Gills chambers.

Type locality. Argentina, Buenos Aires Province, La Plata, Canal Villa Elisa (34 $50^{\circ}$ 'S-57 $\left.59^{\prime} \mathrm{W}\right)$.

Paratypes. 3 and 2 , MLP-Cr 26013, MLP-Cr 26014, 1988-09-12, same locality as type. Collector: Schuldt, M

Current status. Valid species.

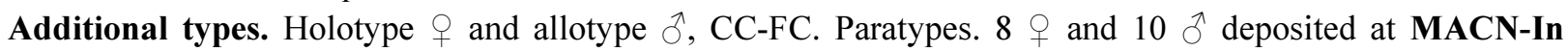
33654. 1982-11-12. Same collector as paratypes.

Reference. Verdi \& Schuldt (1988).

Order Amphipoda Latreille, 1816

Suborder Lowry \& Myers, 2013

Family Dogielinotidae Gurjanova, 1953

Hyalella misionensis Colla \& César, 2015

Type locality. Argentina, Misiones Province, Yabotí Biosphere Reserve, San Pedro and Guaraní Departments, Salto Isipós, near Paraíso stream $\left(27^{\circ} 13.19^{\prime} \mathrm{S}-54^{\circ} 02.73^{\prime} \mathrm{W}\right)$.

Holotype. ${ }^{\lambda}$, MLP-Cr 26978, 2011-10-19. Collectors: César, I and Martin, S.M.

Paratypes. $15 \AA, 15+7$ ovigerous + , and 70 juveniles, MLP-Cr 26979, same locality, date and collectors as type.

Current status. Valid species.

Reference. Colla César (2015).

Suborder Gammaridae Latreille, 1802

Family Uristidae Hurley, 1963

Uristes yamana Chiesa \& Alonso de Pina, 2007

Type locality. Argentina, Tierra del Fuego Province, Beagle Channel, Isla Lucas, (5450'S$\left.68^{\circ} 19^{\prime} \mathrm{W}\right)$.

Paratypes. 1 immature $\widehat{\text { O }}$, MLP-Cr 26111, same locality as type, 1997-12-10. Collector: Lovrich, G.; 3 unknown sex, MLP-Cr 26112, same locality as type, 1996-10-27. Collector: Roccatagliata, D.

Current status. Valid species.

Additional types. Holotype. ovigerous female MACN-In 37099. Paratypes. 2 immature $\widehat{\partial}, 1$ immature ${ }_{+}, 3$ unknown sex, MACN-In 37100; 1 ovigerous + , MACN-In 37101.

Reference. Chiesa \& Alonso de Pina (2007). 
Family Mysidae Haworth, 1825

Mysidopsis rionegrensis Hoffmayer, 1993

Type locality. Argentina, Chubut Province, San Matías Gulf $\left(41^{\circ} 00^{\prime} \mathrm{S}-65^{\circ} 06^{\prime} \mathrm{WL}\right)$.

Holotype. $\widehat{\sigma}$, MLP-Cr 26915. 1968-3-5. Collector: Hoffmayer, M. S.

Allotype. + , MLP-Cr 26916, same locality and collector as type.

Paratypes. 11 ô, 2 , MLP-Cr 26917, same locality, date and collector as type.

Current status. Valid species.

Additional types. Paratypes USNM.

Reference. Hoffmayer (1993).

*Pseudobranchiomysis arenae Carcedo, Fiori \& Hoffmeyer, 2013

Type locality. Argentina, Buenos Aires Province, Monte Hermoso County. (38 $\left.59^{\prime} \mathrm{S}-61^{\circ} 06^{\prime} \mathrm{W}\right)$.

Holotype. ${ }^{\wedge}$, MLP-Cr 26847, 2010-11-25. Collector : Carcedo C.

Allotype. $q$, MLP-Cr 26848-1, same locality, date and collector as type.

Paratypes. $2 \delta$ and 2 , MLP-Cr 26848-2, same locality, date and collector as type.

Current status. Valid species.

Reference. Carcedo et al. (2013).

Superorder Eucarida Calman, 1904

Order Decapoda Latreille, 1802

Suborder Pleocyemata Burkenroad, 1963

Infraorder Anomura MacLeay, 1838

Family Aeglidae Dana, 1852

Aegla intercalata Bond-Buckup \& Buckup, 1994

Type locality. Argentina, Catamarca Province, Las Lajas River $\left(28^{\circ} 13^{\prime} \mathrm{S}-65^{\circ} 52^{\prime} \mathrm{W}\right)$.

Holotype. $\widehat{o}$ MLP-Cr 46/1, 1959-04. Collector: Sorrays, L.P.

Paratypes. $35 \AA$ and 11 , MLP-Cr 46/2, same locality, date and collector as type; 7 unknown sex. MLP-Cr

01/1, Argentina, Tucumán, Quebrada de los Sosas, Camino a Tafí del Valle, 1960-11-09.

Current status. Valid species.

Additional types. Paratypes MACN-In 7812, MACN-In 9418 and FML 0.0027.

Reference. Bond-Buckup \& Buckup (1994), César \& Damborenea (2010).

\section{Aegla montana Ringuelet, 1960}

Type locality. Argentina, Mendoza Province, El Sosneado, Atuel River ( $\left.35^{\circ} 04^{\prime} \mathrm{S}-69^{\circ} 34^{\prime} \mathrm{W}\right), 1543 \mathrm{~m}$ a.s.l.

Syntypes. 5 $\curvearrowright$, MLP-Cr 41 (other numbers 86 and 4815), 1921. Collector: Carette, C.

Current status. Junior synonym of Aegla affinis Schmitt, 1942 according to Bond-Buckup \& Buckup (1994).

Remarks. Ringuelet (1960) described the holotype and 4 paratypes. The collection presents only one lot with five specimens.

Reference. Ringuelet (1960), Bond-Buckup \& Buckup (1994) and César \& Damborenea (2010). 
Type locality. Argentina, Mendoza Province, Fray Luis Beltrán $\left(33^{\circ} 01^{\prime} \mathrm{S}-68^{\circ} 39^{\prime} \mathrm{W}\right)$.

Lectotype. ${ }^{\lambda}$, MLP-Cr 87.

Paralectotypes: $\mathbf{1 3} \widehat{\overbrace{}}$ and $\mathbf{1 9}$, , same locality as type, 1942-02. Collector: McDonagh, E.

Current status. Valid species.

Remarks. Ringuelet (1948b: 315) reported the type material, 19 $\delta$ and 24 $\odot$, as "cotypes", a term, which is not recognized by the International Code of Zoological Nomenclature (Recommendation 73E). Bond-Buckup \& Buckup (1994) designated lectotype and paralectotypes.

Reference. Ringuelet (1948b), Bond-Buckup \& Buckup (1994) and César \& Damborenea (2010).

\section{Aegla septentrionalis Bond-Buckup \& Buckup, 1994}

Type locality. Bolivia, Tupiza $\left(21^{\circ} 26^{\prime} 60^{\prime \prime} \mathrm{S}-65^{\circ} 43^{\prime} 00^{\prime \prime} \mathrm{W}\right)$.

Holotype. $\widehat{\jmath}$, MLP-Cr 29/2 (other number 53), 1948. Collector: Klepovich, P.

Paratypes. $3 \AA$ and $1+$ MLP-Cr 29/1 (other number 53), same locality, date and collector as type; MLP-Cr 104/2, Bolivia, Tarija, Río Sella ( $\left.21^{\circ} 28^{\prime} 13,60^{\prime \prime} \mathrm{S}-64^{\circ} 44^{\prime} 42,9^{\prime \prime} \mathrm{W}\right)$ (200 m ahead the mouth of Guadalquivir River);

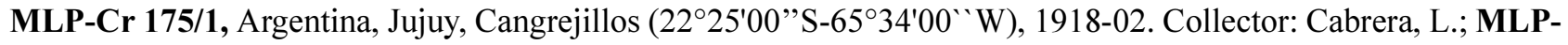
Cr 79, Argentina, Salta, Río Arenales, 1946-05-05. Collector: Mac Donagh, E.

Current status. Valid species.

Reference. Bond-Buckup \& Buckup (1994), César \& Damborenea (2010).

\section{Aegla singularis Ringuelet, 1948}

Type locality. Argentina, Misiones Province, Pindapoy County $\left(27^{\circ} 45^{\prime} \mathrm{S}-55^{\circ} 48^{\prime} \mathrm{W}\right)$.

Syntype. $2 \bigcirc$ and 3 , MLP-Cr 109 (other number 148), 1943-06-05. Collector: Bridarolli, A.

Current status. Valid species.

Reference. Ringuelet (1948c), Bond-Buckup \& Buckup (1994), César \& Damborenea (2010).

\section{References}

Alarcos, A.J. \& Timi, J.T. (2011) A new species of Acanthochondria (Copepoda: Chondracanthidae) parasitizing the flounder Xystreurys rasile (Pleuronectiformes: Paralichthyidae) from Argentina. Folia Parasitologica, 58, 164-168. https://doi.org/10.14411/fp.2011.016

Belk, D. \& Brtek, J. (1995) Checklist of the Anostraca. Hydrobiologia, 298, 315-353. https://doi.org/10.1007/BF00033826

Birabén, M. (1946) Sobre tres nuevas especies de Branchinecta de la Patagonia (Phyllopoda). Notas Museo La Plata, Zoología, 11(94), 137-139.

Birabén, M. (1951) Nuevo género de Phyllopoda Anostraca (Crust.). Physis, 20, 324-329.

Bond-Buckup, G. \& Buckup, L. (1994) A Familia Aeglidae (Crustacea, Decapoda, Anomura). Arquivos de Zoologia, 32, 176179. https://doi.org/10.11606/issn.2176-7793.v32i4p159-346

Braicovich, P.E. \& Timi, J.T. (2009) Acanthochondria serrani sp. n. (Copepoda: Chondracanthidae) parasitic on Serranus auriga (Perciformes: Serranidae) from Argentinean waters. Folia Parasitologica, 56, 313-316. https://doi.org/10.14411/fp.2009.036

Cantatore, D.M.P. \& Timi, J.T. (2010) Acanthochondria helicoleni sp. nov. (Copepoda, Chondracanthidae) parasitic on Helicolenus lahillei (Scorpaeniformes, Sebastidae) from Argentinean waters. Acta Parasitologica, 55, 386-391. https://doi.org/10.2478/s11686-010-0052-0

Cantatore, D.M.P., Lanfranchi, A.L. \& Timi, J.T. (2011) New species of Acanthochondria (Copepoda: Chondranthidae) infecting the long tail southern cod, Patagonotothen ramsayi (Perciformes: Nototheniidae), from Patagonian waters, Argentina. Journal of Parasitology, 97, 1007-1011. https://doi.org/10.1645/GE-2815.1

Carcedo, C., Fiori S. \& Hoffmeyer, M. (2013) Pseudobranchiomysis arenae, a new genus and species of Leptomysinae 
(Crustacea: Mysida) in Argentinian sandy beaches. Zootaxa, 3647 (3), 479-487.

https://doi.org/10.11646/zootaxa.3647.3.5

César, I.I. (1985) Branchinecta achalensis sp. nov. (Crustacea: Anostraca) de La Pampa de Achala (Córdoba, República Argentina). Neotropica, 31, 89-100.

César, I.I. (1987a) Branchinecta leonensis sp. nov. (Crustacea: Anostraca) de la provincia de Santa Cruz, República Argentina. Notas Museo La Plata, Zoología, 21 (206), 77-86.

César, I.I. (1987b). Branchinecta santacrucensis sp. nov. (Crustacea: Anostraca) de la Patagonia. Neotropica, 33 (90), $75-82$.

César, I.I. (1991) Limnadia santiaguensis, sp. n. (Conchostraca, Limnadiidae) de la Argentina. Iheringia, Serie Zoología, 71 , 99-110.

César, I.I. \& Damborenea, C. (2010) Type and non-type specimens of Aegla (Decapoda: Anomura: Aeglidae) housed in the Museo de La Plata, Argentina. Zootaxa, 2337, 31-46.

Chiesa, I.L. \& Alonso de Pina, G.M. (2007) A new species of Uristes Dana, 1849 (Amphipoda: Lysianassoidea: Uristidae) from the Beagle Channel, Argentina. Proceedings of the Biological Society of Washington, 120, 446-458. https://doi.org/10.2988/0006-324X(2007)120[446:ANSOUD]2.0.CO;2

Cohen, G.R. (1981) Notas sobre Anostracos Neotropicales (Crustacea).I. Branchinecta valchetana sp. nov. y redescripción de Branchinecta palustris Birabén, 1946. Physis, B40 (98), 1-13.

Cohen, G.R. (1995) Concerning the identity of Branchinecta santacrucensis César, 1987. Hydrobiologia, 298, $239-240$. https://doi.org/10.1007/BF00033817

Colla, M.F. \& César, I.I. (2015) A new species of Hyalella (Crustacea, Amphipoda, Dogielinotidae) from the Atlantic Forest of Misiones, Argentina. Zookeys, 481, 25-38. https://doi.org/10.3897/zookeys.481.9037

Díaz, A.R. \& Lopretto, E.C. (2009) A new species of Cypriconcha Sars (Crustacea: Ostracoda) from high mountain wetlands of Argentina. Zootaxa, 2291, 51-64.

Díaz, A.R. \& Lopretto, E.C. (2011) A new species of Keysercypria Karanovic (Crustacea: Ostracoda) from Argentina. Zootaxa, 3063, 64-68.

Díaz, A.R. \& Martens, K. (2014) On Argentocypris sara gen. nov., sp. nov. (Ostracoda) from the Patagonian wetlands of Argentina. Crustaceana, 87, 507-550. https://doi.org/10.1163/15685403-00003300

Doti, B.L. \& Roccatagliata, D. (2005) On two paramunnid species from the Beagle Channel, Argentina (Crustacea: Isopoda: Asellota). Proceedings of the Biologial Society of Washington, 118, 509-521. https://doi.org/10.2988/0006-324X(2005)118[509:OTPSFT]2.0.CO;2

Etchegoin, J.A., Timi, J.T. \& Sardella, N.H. (2003) Argentinochondria patagonensis n. gen., n. sp. (Copepoda: Chondracanthidae) parasitic on Genypterus brasiliensis (Pisces: Ophidiidae) from Patagonia, Argentina. Journal of Parasitology, 89, 701-704.

https://doi.org/10.1645/GE-3110

Etchegoin, J.A., Timi, J.T. \& Lanfranchi, A.L. (2006) Redescription of Neobrachiella spinicephala (Ringuelet, 1945) parasitic on Pinguipes brasilianus Cuvier, 1829 from Argentina, with the first description of the male. Acta Parasitologica, 51, 290-293. https://doi.org/10.2478/s11686-006-0044-2

Frey, D.G. (1993) Species of Pleuroxus (Anomopoda, Chydoridae) from the subantarctic islands and southernmost South America: a partial unravelling of the Pleuroxus aduncus problem. Hydrobiologia, 262, 145-188. https://doi.org/10.1007/BF00010882

Gamarra-Luques, C.D., Vega I.A., Koch, E. \& Castro-Vazquez, A. (2004) -Intrahost distribution and trasmission of a new species of cyclopoid copepod endosymbiotic to a freshwater snail Pomacea canaliculata (Caenogastropoda, Ampullariidae) from Argentina. Biocell, 28, 155-164.

Hoffmeyer, M.S. (1993) Mysidopsis rionegrensis, a new species of Mysidacea from San Matias Gulf, Argentina. Physis, 48, 15-19.

Just, J. \& Wilson, G.D.F. (2007) Revision of Austrosignum Hodgson and Munnogonium George \& Strömberg (Paramunnidae) with descriptions of eight new genera and two new species, (Crustacea: Isopoda: Asellota). Zootaxa, 1515, 1-29.

Martin, J.W. \& Davis, G.E. (2001) An updated Classification of the Recent Crustacea. Science Series 39. Natural History Museum of Los Angeles County, Los Angeles, $132 \mathrm{pp}$.

Pereira, A.N., Timi, J.T., Lanfranchi, A.L. \& Luque, J.L. (2012) A new species of Colomatus (Copepoda, Phylichthyidae) parasitic on Mullus argentinae (Perciformes, Mullidae) from South American Atlantic coast. Acta Parasitologica, 57, 323-328. https://doi.org/10.2478/s11686-012-0032-7

Pessacq, P., Epele, L.B. \& Rogers, C.D. (2011) A new species of Lynceus (Crustacea: Branchiopoda: Laevicaudata) from Patagonia, with comments on laevicaudatan systematic. Zootaxa, 3043, 25-32.

Ramírez, F.C. (1967) Ostrácodos de lagunas de la provincia de Buenos Aires. Revista del Museo de La Plata, Nueva Serie, Zoología, 73 (10), 5-54.

Ringuelet, R.A. (1943) Revisión de los argúlidos Argentinos (Crustácea, Branchiura) con el catálogo de las especies neotropicales. Revista del Museo de La Plata, Nueva Serie, Zoología, 3(19), 43-99. 
Ringuelet, R.A. (1945) Parabrachiella spinicephala nueva especie de copépodo parasito. Notas del Museo de La Plata, Zoología, 10 (86), 129-135.

Ringuelet, R.A. (1948a) Argúlidos del Museo de La Plata. Revista del Museo de La Plata, Nueva Serie, Zoología, 5, $281-296$.

Ringuelet, R.A. (1948b) Los" cangrejos" argentinos del genero Aegla de Cuyo y la Patagonia. Revista del Museo de La Plata, Nueva Serie, Zoología, 5, 315-319.

Ringuelet, R.A. (1948c) Una nueva Aegla del Nordeste argentino. Notas del Museo de La Plata, Zoología, 13 (111), $203-208$.

Ringuelet, R.A. (1960) Notas sobre "Aegla" de Argentina y Paraguay (Crustacea, Decapoda, Anomura). Physis, 21 (61), $231-$ 239.

Timi, J.T. \& Etchegoin, J.A. (1996) A new species of Lernanthopus (Copepoda: Lernanthropidae) parasite of Cynoscionstriatus (Pisces: Sciaenidae) from Argentinean waters, and new records of Lernanthropus trachuri. Folia Parasitologica, 43, 7174.

Timi, J.T. \& Sardella, N.H. (1997) New species of Nothobomolochus (Copepoda: Bomolochidae) parasitic on Engraulis anchoita (Pisces: Engraulidae) from Argentina. Folia Parasitologica, 44, 305-308.

Timi, J.T., Etchegoin, J.A \& Lanfranchi, A.L. (2004) Blias marplatensis sp. nov. (Copepoda, Chondracanthidae). Acta Parasitologica, 49, 332-335.

Verdi, A.C. \& Schuldt, M. (1988) Descripción y biología de Probopyrus ringueleti n. sp. (Crustacea, Bopyridae). Efectos sobre el hospedador Palaemonetes argentinus Nobili (Crustacea, Caridea, Palaemonidae). Anales de la Sociedad Científica Argentina, 118, 15-26. 